\title{
Correction to: LIGHTAWE_case studies of LIGHT spreAd in poWder materials: a montE carlo simulation tool for research and educational purposes
}

\section{Panagiotis Liaparinos ${ }^{1}$}

Published online: 28 November 2019

(c) Springer-Verlag GmbH Germany, part of Springer Nature 2019

Due to a technical error during the production process, in the originally published online PDF version of this Article part of Appendix B was not published. This error has now been corrected. The Publisher apologizes for this mistake and all related inconveniences caused by this.

The original article can be found online at https://doi.org/10.1007/ s00340-019-7267-z.

Panagiotis Liaparinos

liapkin@uniwa.gr

1 Radiation Physics, Materials Technology and Biomedical

Imaging Laboratory, Department of Biomedical

Engineering, University of West Attica, Athens, Greece 


\section{Introduction}

Research and development of imaging systems can be looked at from two different perspectives: (a) the purpose of image acquisition and (b) the physical principles and technological aspects of the system. For instance, in medical imaging, new innovations are associated either with the radiographer's option and assessment of a particular patient examination for validate diagnosis or with the physical and technological factors that affect the imaging chain and contribute to image formation of improved quality [1]. Regarding the second point of view, one crucial step of the so-called "image chain approach" that lead to the final output (i.e., the image) is the fidelity of the information transfer processes [2,3]. A variety of imaging techniques with tremendous potential of applications use the branch of light transfer mechanisms with purpose to construct high-performance displays and devices in the visible portion of the electromagnetic spectrum [4-10]. More specifically, intermediate step of the imaging system involves the behavior and properties of light, including its interactions with matter. There are cases where the state of matter (either organic or inorganic) is found in granular structure, that is, as in the form of an ensemble of particles. In such powder materials, subject of interest and examination is the trajectories of light ray tracks, the type of light beam interactions with the particles and the overall optical transmission, reflection and diffusion.

This realm of opportunity for knowledge discovery in imaging information and technology can be still thought of a Pandora's Box since comprehensive quantitative and qualitative understanding is lacking and experimental modalities are extremely costly for such demands. On the other hand, the introduction of computational techniques, the so-called Monte Carlo methods [11], and their widespread use [12, 13], on radiation transport simulations over the last 50 years tender them very promising to solve problems that might be deterministic in principle, however, is difficult or impossible to use other approaches. In particular, the advantage of Monte Carlo method to perform experiments that would be otherwise impossible could be proved crucial for the enhancement of research curiosity which in turn may lead to spectacular scientific discoveries. In this paper, LIGHTAWE Monte Carlo simulation tool is introduced and presented with purpose to insight and further enlighten the study of light spread in powder materials for either research or educational applications. The algorithms of LIGHTAWE are based on Mie scattering theory and Henyey-Greenstein distribution [14-17]. The main issues of LIGHTAWE (design and capabilities) are analytically addressed and described below in terms of the: (i) physics embedded, (ii) treatment of the software, (iii) input data contents (iv) simulation options, (v) output results, (vi) advantages and benefits and (vii) disadvantages and limitations.

\section{Materials and methods}

The LIGHTAWE Monte Carlo simulation tool (software as well as corresponding support-if required) is sent to the applicant via e-mail. The main components are briefly presented below including the: (a) physics, and (b) treatment of LIGHTAWE Monte Carlo software.

\subsection{The physics of LIGHTAWE}

The purpose of LIGHTAWE is to provide optical diffusion studies in granular materials. The powder material was considered as an ensemble of uniformly distributed spherical particles glued together with a binding material and coated in three-dimensional layer. Light quanta are generated and thereafter diffused within the material. Light attenuation is described through light ray interactions (scattering and absorption) with the particles which play the role of "optical scatterers" within the granular structure. A light photon history is considered to start within the phosphor layer with the coordinates of the initial position and the initial direction angles (polar and azimuthal) determined by an isotropic distribution. Thereafter, the intrinsic processes of light propagation are summarized in three stages: (i) first stage: the light photon travels a free path length and afterwards interacts with a phosphor particle, (ii) second stage: the light photons is either absorbed or scattered and finally (iii) third stage: the trajectory of the light photon is terminated (in case of absorption) or the direction of the light photon is changed (in case of scattering). The mathematical expressions of the light quanta interaction mechanisms are analytically provided in a previous article (Appendix B of Ref. [18]). In addition, mathematical analysis of the simulation algorithms requires the evaluation of three optical parameters which correspond to the aforementioned stages: (a) the light extinction coefficient, $m_{\mathrm{ext}}$ and (b) the anisotropy scattering factor, $g$ and (c) the percentage light absorption probability $p$. Their evaluation is carried out within the framework of Mie scattering theory and Henyey-Greenstein distribution, as analytically provided in a previous article (Appendix A of Ref. [18]).

\subsection{The treatment of LIGHTAWE}

LIGHTAWE has been developed in MATLAB environment and includes a user-friendly guide in the command window divided in two branches: (a) The input data of the desired simulation and (b) The output results of the executable simulation. 
Table 1 Table summarizes the customization of LIGHTAWE Monte Carlo software

\begin{tabular}{|c|c|c|}
\hline \multicolumn{3}{|l|}{ LIGHTAWE Monte Carlo software } \\
\hline \multicolumn{3}{|c|}{$\begin{array}{l}\text { Required condition: } 0.1<x<100 \text { where } x=\pi d n_{\text {medium }} / \lambda, x \text { : Mie size parameter, } d \text { : particle diameter, } n_{\text {medium }} \text { : refractive index of the of the medium, } \lambda \text { : light wave- } \\
\text { length }\end{array}$} \\
\hline \multicolumn{3}{|l|}{ Layer settings } \\
\hline$x$-dimension $(\mathrm{cm})$ & $0.1-10$ & \\
\hline$y$-dimension $(\mathrm{cm})$ & $0.1-10$ & \\
\hline Thickness $(\mu \mathrm{m})$ & $1-1000$ & \\
\hline Number of pixels & $64-512$ & \\
\hline \multicolumn{3}{|l|}{ Structural properties } \\
\hline Particle diameter (nm) & $10-20,000$ & \\
\hline Packing density (\%) & $10-90$ & \\
\hline \multicolumn{3}{|l|}{ Optical properties } \\
\hline Light wavelength (nm) & $400-700$ & \\
\hline Refractive index of the material (Real part) & $1.5-2.5$ & \\
\hline Refractive index of the material (Imaginary part) & $10^{-6}-10^{-4}$ & \\
\hline Refractive index of the medium (binder) & $1.0-1.5$ & \\
\hline \multicolumn{3}{|l|}{ Light beam properties } \\
\hline Number of light photons & $1-10^{7}$ & \\
\hline \multicolumn{3}{|l|}{ Simulation options } \\
\hline \multicolumn{3}{|l|}{ (a) Fixed light point source } \\
\hline Site of light source (\% layer depth) & $10-90$ & \\
\hline \multicolumn{3}{|c|}{ (b) Distribution of light sources obtained by $\mathrm{X}$-ray attenuation } \\
\hline $\mathrm{X}$-ray energy $(\mathrm{keV})$ & $10-100$ & \\
\hline Material composition & Table 2 & \\
\hline Material density $\left(\mathrm{g} / \mathrm{cm}^{3}\right)$ & Table 2 & \\
\hline \multicolumn{3}{|c|}{ Recommended values apart from the fixed X-ray energy range } \\
\hline \multicolumn{3}{|l|}{ Output data } \\
\hline \multirow[t]{3}{*}{ Optical parameters } & The light extinction coefficient mext $(\mu \mathrm{m})^{-1}$ & \\
\hline & The anisotropy factor $g$ & \\
\hline & The light absorption probability $p(\%)$ & \\
\hline Computation time (min) & According to the setting parameters & \\
\hline \multirow[t]{2}{*}{ Figure } & Light performance (reflected, transmitted, absorbed) & LSF (reflected, transmitted) \\
\hline & Distribution of light photons per depth of interaction & MTF (reflected, transmitted, pixel) \\
\hline
\end{tabular}

\subsubsection{The input data of LIGHTAWE}

The input data required to feed the software of LIGHTAWE are summarized in Table 1. Initially, the user browses for the folder of the code in the MATLAB platform, thereafter the name LIGHTAWE is typed in the command window and the execution of the simulation starts. Basic required condition for the execution of proposed simulations is the Mie size parameter (Table 1) to be more than 0.1 and less than 100 (the so-called "Mie regime"). In such case, the following data are asked from the user: (i) Layer settings: $x$ dimension, $y$ dimension, thickness and number of pixels, (ii) Structural properties: Particle diameter and Packing density, (iii) Optical properties: light wavelength, particle refractive index (real part), particle refractive index (imaginary part), binder refractive index. The numerical values of the aforementioned parameters, provided in Table 1, correspond to representative typical values, however, are recommended (suggestive) for the decision of user's choice (apart from the X-ray energy range which is fixed). A point worth commenting is that the selection of particular parameters affect correspondingly the computation time.

\subsubsection{The simulation options of LIGHTAWE}

After inserting the input data described above, the user sets the number of the light photons used in the proposed simulation. Thereafter, there are two main options of simulations embedded in LIGHTAWE: (a) Fixed light point source (i.e., fixed depth of light photon generation within the layer) and (b) Distribution of light sources obtained from X-ray attenuation (i.e., variable depth of optical photon generation within the layer taken from the penetration of an X-ray beam of particular energy within a specific phosphor material). The first case examines in general the light spread in powder materials (including phosphors) while the second case examines the light spread in specific powder phosphor materials. Therefore, the first case requires the percentage 
Table 2 List of phosphor materials included in LIGHTAWE

\begin{tabular}{|c|c|c|c|c|c|c|}
\hline \multicolumn{2}{|c|}{ Phosphor materials } & \multirow{2}{*}{$\begin{array}{l}\text { Typical } \\
\text { density }(\mathrm{g} / \\
\left.\mathrm{cm}^{3}\right)\end{array}$} & \multirow{2}{*}{$\begin{array}{l}\text { Probability of photo- } \\
\text { electric effect for X-ray } \\
\text { energies } 10 \mathrm{keV} \text { and } \\
100 \mathrm{keV}\end{array}$} & \multirow{2}{*}{$\begin{array}{l}\text { Fluorescence yield } \\
\text { values } \omega_{\mathrm{K}} \text { of the heaviest } \\
\text { element }\end{array}$} & \multirow{2}{*}{$\begin{array}{l}\text { Mean Energy of } K \alpha \\
\text { X-rays of the heaviest } \\
\text { element }(\mathrm{keV})\end{array}$} & \multirow{2}{*}{$\begin{array}{l}\text { Mean path length of } K \\
\text { X-rays of the heaviest } \\
\text { element }(\mu \mathrm{m})\end{array}$} \\
\hline 1 & $\mathrm{Ba}_{4} \mathrm{Ce}_{3} \mathrm{~F}_{17}$ & & & & & \\
\hline 2 & $\mathrm{BaCl}_{2}$ & 3.89 & $0.9993-0.9185$ & 0.8997 & 32.00 & 831 \\
\hline 3 & $\mathrm{BaF}_{2}$ & 4.90 & $0.9993-0.9314$ & 0.8997 & 32.00 & 617 \\
\hline 4 & $\mathrm{BaHfO}_{3}$ & 8.40 & $0.9994-0.9601$ & 0.9505 & 55.20 & 389 \\
\hline 5 & $\mathrm{BaLu}_{2} \mathrm{~F}_{8}$ & 7.00 & $1.0000-0.9568$ & 0.9487 & 53.52 & 578 \\
\hline 6 & $\mathrm{BaThF}_{6}$ & 6.70 & $0.9842-0.9221$ & 0.9691 & 91.65 & 1564 \\
\hline 7 & $\mathrm{BaY}_{2} \mathrm{~F}_{8}$ & 5.00 & $0.9989-0.8756$ & 0.8997 & 32.00 & 399 \\
\hline 8 & $\mathrm{Ba}_{4} \mathrm{Y}_{3} \mathrm{~F}_{17}$ & 5.25 & $1.0000-0.9040$ & 0.8997 & 32.00 & 437 \\
\hline 9 & $\mathrm{Bi}_{4} \mathrm{Ge}_{3} \mathrm{O}_{12}(\mathrm{BGO})$ & 7.10 & $0.9993-0.9712$ & 0.9643 & 75.96 & 1315 \\
\hline 10 & $\mathrm{CaHfO}_{3}$ & 7.50 & $1.0000-0.9574$ & 0.9505 & 55.20 & 891 \\
\hline 11 & $\mathrm{CaWO}_{4}$ & 6.10 & $0.9990-0.9579$ & 0.9538 & 58.65 & 1236 \\
\hline 12 & $\mathrm{CeCl}_{3}$ & 3.90 & $0.9993-0.9143$ & 0.9096 & 34.50 & 1007 \\
\hline 13 & $\mathrm{CeF}_{3}$ & 6.20 & 0.9993-0.9309 & 0.9096 & 34.50 & 578 \\
\hline 14 & $\mathrm{CsF}$ & 4.10 & $1.0000-0.9370$ & 0.8942 & 30.80 & 625 \\
\hline 15 & CsI & 4.51 & $0.9994-0.9434$ & 0.8942 & 30.80 & 526 \\
\hline 16 & $\mathrm{CsGd}_{2} \mathrm{~F}_{7}$ & 5.50 & $1.0000-0.9462$ & 0.9320 & 42.65 & 464 \\
\hline 17 & $\mathrm{Cs}_{2} \mathrm{LiYCl}_{6}$ & 3.30 & $0.9991-0.8938$ & 0.8942 & 30.80 & 730 \\
\hline 18 & $\mathrm{Cs}_{3} \mathrm{LuCl}_{6}$ & 3.80 & $0.9993-0.9404$ & 0.9487 & 53.52 & 773 \\
\hline 19 & $\mathrm{Cs}_{3} \mathrm{Lu}_{2} \mathrm{Cl}_{9}$ & 4.00 & $0.9992-0.9245$ & 0.9487 & 53.52 & 1113 \\
\hline 20 & $\mathrm{Cs}_{2} \mathrm{NaCeCl}_{6}$ & 3.20 & $1.0000-0.8712$ & 0.9096 & 34.50 & 1667 \\
\hline 21 & $\mathrm{Cs}_{2} \mathrm{NaLaCl}_{6}$ & 3.20 & $0.9993-0.9138$ & 0.9049 & 33.24 & 1164 \\
\hline 22 & $\mathrm{Cs}_{2} \mathrm{NaLuCl}_{6}$ & 3.70 & $0.9993-0.9369$ & 0.9487 & 53.52 & 944 \\
\hline 23 & $\mathrm{GdAlO}_{3}(\mathrm{GAP})$ & 7.50 & $1.0000-0.9431$ & 0.9320 & 42.65 & 656 \\
\hline 24 & $\mathrm{GdBr}_{3}$ & 4.60 & $1.0000-0.9267$ & 0.9320 & 42.65 & 689 \\
\hline 25 & $\mathrm{GdBO}_{3}$ & 6.30 & $0.9995-0.9473$ & 0.9320 & 42.65 & 736 \\
\hline 26 & $\mathrm{Gd}_{3} \mathrm{Ga}_{5} \mathrm{O}_{12}$ & 7.10 & $0.9993-0.9244$ & 0.9320 & 42.65 & 638 \\
\hline 27 & $\mathrm{Gd}_{2} \mathrm{O}_{2} \mathrm{~S}(\mathrm{GOS})$ & 7.30 & $0.9996-0.9551$ & 0.9320 & 42.65 & 554 \\
\hline 28 & $\mathrm{Gd}_{2} \mathrm{SiO}_{5}(\mathrm{GSO})$ & 6.70 & $0.9995-0.9484$ & 0.9320 & 42.65 & 673 \\
\hline 29 & $\mathrm{Gd}_{3} \mathrm{Sc}_{2} \mathrm{Al}_{3} \mathrm{O}_{12}$ & 5.60 & 0.9994-0.9309 & 0.9320 & 42.65 & 996 \\
\hline 30 & $\mathrm{~K}_{2} \mathrm{LaCl}_{5}$ & 2.90 & $0.9991-0.8592$ & 0.9049 & 33.24 & 1663 \\
\hline 31 & $\mathrm{LaBO}_{3}$ & 5.20 & $0.9993-0.9256$ & 0.9049 & 33.24 & 674 \\
\hline 32 & $\mathrm{LaB}_{3} \mathrm{O}_{6}$ & 4.20 & $0.9990-0.8969$ & 0.9049 & 33.24 & 1108 \\
\hline 33 & $\mathrm{LaBr}_{3}$ & 5.30 & $1.0000-0.9045$ & 0.9049 & 33.24 & 334 \\
\hline 34 & $\mathrm{LaCl}_{3}$ & 3.90 & 0.9993-0.9094 & 0.9049 & 33.24 & 963 \\
\hline 35 & $\mathrm{LaF}_{3}$ & 5.90 & $0.9993-0.9275$ & 0.9049 & 33.24 & 586 \\
\hline 36 & $\mathrm{LaLuO}_{3}$ & 8.40 & $0.9994-0.9594$ & 0.9487 & 53.52 & 349 \\
\hline 37 & $\mathrm{LiBaF}_{3}$ & 5.30 & $0.9992-0.9201$ & 0.8997 & 32.00 & 650 \\
\hline 38 & $\mathrm{LiLuF}_{4}$ & 6.00 & $0.9993-0.9569$ & 0.9487 & 53.52 & 1087 \\
\hline 39 & $\mathrm{LiLuSiO}_{4}$ & 5.50 & $1.0000-0.9528$ & 0.9487 & 53.52 & 1247 \\
\hline 40 & $\mathrm{LiYF}_{4}$ & 4.00 & $0.9976-0.7452$ & 0.7155 & 14.92 & 382 \\
\hline 41 & $\mathrm{LuAlO}_{3}(\mathrm{LuAP})$ & 8.30 & $0.9994-0.9582$ & 0.9487 & 53.52 & 760 \\
\hline 42 & $\mathrm{Lu}_{3} \mathrm{Al}_{5} \mathrm{O}_{12}$ & 6.70 & $0.9993-0.9516$ & 0.9487 & 53.52 & 1057 \\
\hline 43 & $\mathrm{Lu}_{2} \mathrm{O}_{3}$ & 9.40 & $0.9995-0.9684$ & 0.9487 & 53.52 & 544 \\
\hline 44 & $\mathrm{LuBO}_{3}$ & 6.90 & $1.0000-0.9611$ & 0.9487 & 53.52 & 863 \\
\hline 45 & $\mathrm{LuBr}_{3}$ & 4.80 & $1.0000-0.9433$ & 0.9487 & 53.52 & 1066 \\
\hline 46 & $\mathrm{LuCl}_{3}$ & 4.00 & $1.0000-0.9531$ & 0.9487 & 53.52 & 1688 \\
\hline 47 & $\mathrm{LuF}_{3}$ & 8.30 & 0.9994-0.9620 & 0.9487 & 53.52 & 711 \\
\hline
\end{tabular}


Table 2 (continued)

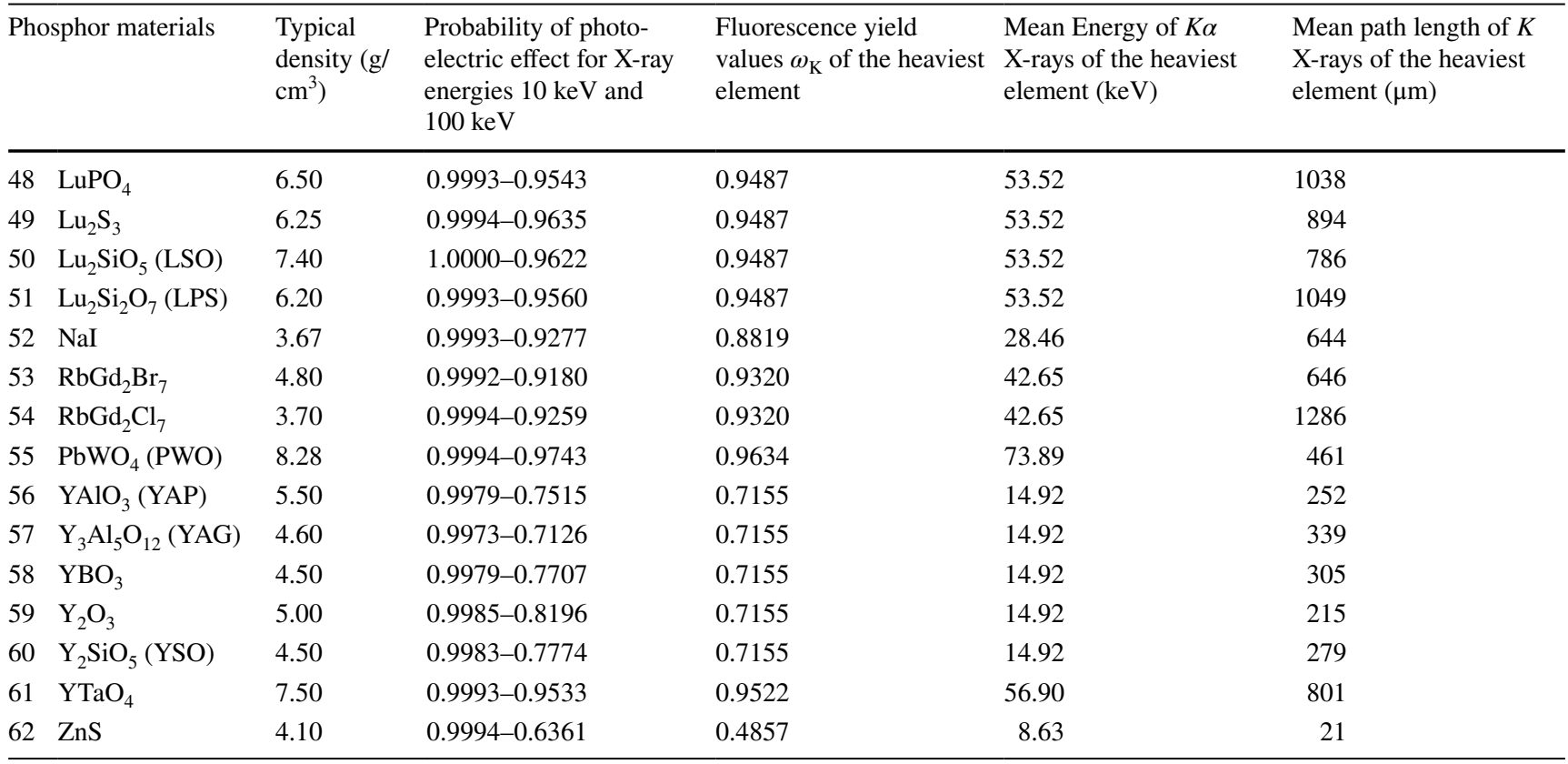

Table provides: (i) the chemical compound, (ii) the typical density of the compound [30-32], (iii) the probability of photoelectric effect for $10 \mathrm{keV}$ and $100 \mathrm{keV}$, (iv) the fluorescence yield values $\omega_{\mathrm{K}}$ of the heaviest element [33], (v) the mean energy of $K \alpha \mathrm{X}$-rays of the heaviest element [34] and (vi) the mean path length of $K \mathrm{X}$-rays of the heaviest element

depth with respect to layer thickness, while the second one, the determination of the X-ray energy $(10-100 \mathrm{keV})$ as well as the chemical compound and the density of the phosphor material.

Regarding the second case, LIGHTAWE includes 62 different chemical compounds with their corresponding density, listed in Table 2. All materials are considered to be in powder form although most of them are commonly and commercially found in other structural forms (e.g., crystal, ceramic, etc.). However, advanced techniques in chemical synthesis methods over the past decade (e.g., chemical vapour deposition, microemulsion route, spray pyrolysis, co-precipitation, sol-gel combustion synthesis, etc.) could replace existing forms of phosphors and give a boost on a new realm of opportunity in granular phosphor development [19] including also powders at submicron and nano scales, even down to $10 \mathrm{~nm}$ since in lower sizes a particle size-dependent dielectric function should be considered (usually assuming a Drude-Sommerfeld model) due to the lattice strain effects [20]. A characteristic example is the cerium doped lutetium orthosilicate- $\mathrm{Lu}_{2} \mathrm{SiO}_{5}$ : $\mathrm{Ce}$ (LSO) fluorescent material which was initially introduced as a single-crystal scintillator [21] but later was also fabricated in powder coating. Table 2 also provides the probability of photoelectric effect for the two limiting values of X-ray energy (10 keV and $100 \mathrm{keV}$ ) to show the domination of photoelectric effect over the scattering (Compton and Rayleigh) phenomena which have been excluded from LIGHTAWE.
To make the code easier and faster, X-ray photons considered to follow only photoelectric absorption and the X-ray energy was considered to be locally absorbed in the interaction site excluding also the emission of fluorescence $\mathrm{X}$-rays ( $K$ X-rays). However, relative additional information of the $K$ $\mathrm{X}$-rays effect is provided in Table 2, such as: (i) the probability of $K \mathrm{X}$-rays production though the fluorescence yield $\omega_{\mathrm{K}}$ of the heaviest element, (ii) the spread of $K \mathrm{X}$-rays within the material through the mean path length of $K \alpha \mathrm{X}$-rays based on their corresponding energy.

\subsubsection{The output results of LIGHTAWE}

The output results of LIGHTAWE are summarized in Table 1. The optical parameters, $m_{\mathrm{ext}}, g$ and $p(\%)$, used to simulate the optical tracks (see Sect. 2.2.1) are provided to the user as output results in the command window. After the termination of the simulation, command window also shows the computation time required to run the proposed simulation and an output figure is observed. The figure illustrates four separate subplots of the following information: (i) the light performance which contains: (a) the percentage of light photons reflected at the upper side of the layer (the back side of the layer also called irradiated side in case of X-ray penetration) (b) the percentage of light photons transmitted at the underside of the layer (the front side of the layer also called non-irradiated side in case of X-ray penetration), and (c) the percentage of light photons absorbed within the material, 
(ii) the distribution of light photons per depth of interaction within the layer, (iii) the Line Spread Function (LSF) for reflection and transmission mode and (iv) the Modulation Transfer Function (MTF) for reflection and transmission mode as well as the so-called pixel-MTF due to the pixel size. The mathematical evaluation of LSF and MTF are analytically provided in a previous article (Appendix C of Ref. [18]). After the execution of each simulation, two additional files have been created within the folder of the code. The first one opens with MATLAB and concerns the illustration of the figure and the second one opens with notepad and records all the setting parameters involved in the simulation. In this manuscript, a specific simulation example of LIGHTAWE is presented in Appendix A (brief physics) and Appendix B (interface). The computation time corresponds to a single computer of i5 CPU $3.47 \mathrm{GHz}$ processor and 4.00 GB RAM.

\section{Discussion}

\subsection{LIGHTAWE advantages and benefits}

LIGHTAWE has been developed to perform dedicated Monte Carlo simulations of light spread in powder materials. An advantage of LIGHTAWE for optical diffusion studies is the separation of two different branches: (i) the study of light spread within a granular layer based only on its structural and optical properties, (ii) the examination of particular powder phosphor materials taking into account the depth of the X-ray penetration. The above options make LIGHTAWE an available and useful tool for a variety of instrumentations which incorporate granular structured materials, such as medical and storage phosphor imaging, radioluminescent and fluorescence sensing, detecting tools of biological structures, and phosphor-based white LED devices. The performance of optical diffusion could also enhance research on particle characterization methods, microscopy techniques, homeland security as well as national defense applications and finally could have an impact on several scientific fields including chemical, energy, optoelectronic and space industries.

The investigation of optical diffusion can be thought of a Pandora's Box since comprehensive quantitative and qualitative understanding is lacking and experimental setups are extremely costly for such demands. However, the option of LIGHTAWE to alter a set of parameters (listed in Table 1) and perform a countless number of probable and comparable simulations, can insight and further enlighten the research of light spread performance. In addition, based on the Monte Carlo methodology, LIGHTAWE can overcome cumbersome analytical modelling, inconvenience and fussiness [22] and can take advantage of computer science to perform experiments that would be otherwise impossible (i.e., the capability to design, evaluate and optimize "virtual" experimental set-ups of "zero cost" or with "low risk" actions) enhancing curiosity-driven future research [23].

An objective disadvantage of Monte Carlo packages is the lack of making user-friendly software with the result the corresponding difficulty of the users to treat the structure of the code. In academic institutions, the majority of $\mathrm{PhD}$ students need particular training on Monte Carlo packages by more experienced researchers or they are called to attend particular workshops [24] to adapt to the setting parameters demands of 'black box' simulation tools. The ease to use treatment of LIGHTAWE is one of its major advantages and can also be a useful computational tool for educational purposes in academic institutions. The study of light spread under the variability of several parameters offers pedagogic ways of learning optical diffusion in powder structures and also provides important information about statistical phenomena that would be impossible to assess otherwise.

\subsection{LIGHTAWE disadvantages and limitations}

Due to the programming algorithms that rely on repeated random sampling to obtain numerical results and reproduce stochastic processes statistically, high computation time is required for precision achievement. Like in most Monte Carlo codes and packages, LIGHTAWE depends on the computation time via the number of light photons and the corresponding simulation of long light tracks. Particular parameters increase dramatically the trajectories of light photons within the material, such as: (i) the increase of the layer thickness, (ii) the increase of the packing density, which expresses the spatial distribution of phosphor particles within the phosphor layer, (iii) the size of the particle diameter (mainly in the submicron scale). However, acceleration of the simulation runs can be accomplished by the availability of advanced calculating tools (e.g., clusters or powerful CPUs). Of course, Monte Carlo is an average method, i.e., the average expectation of one observable is computed, however, this approach is fine if the system is ergodic [25].

LIGHTAWE provides a series of capabilities on studying the optical diffusion, however, light propagation modeling is subject of several limitations. The investigation of light spread in real powder structures is limited to the considerations of X-ray and light beam characteristics, such as (i) the absence of X-ray scattering, $K X$-rays and Auger electrons, polychromatic light photons, reflection at the boundaries, and absorbing dyes incorporation. The modelling of light spread is based on specific methodology (Mie-scattering algorithms) and the accuracy is related to Riccati-Bessel functions. The assumption of spherical particles in contrast to arbitrary sized and shaped particles in real structures is also an issue worth commenting.

The validity of the present computational program has been provided in terms of: (a) the algorithms applied for the sampling 
of physical parameters, such as (i) the free path length, (ii) the optical attenuation (scattering and absorption) coefficients, and (iii) the scattering angles, [26] (b) the absorption and scattering efficiency factors and the complex elements of Mueller scattering matrix $[26,27]$ (c) the prediction and comparison of the optical parameters (light extinction coefficient $m_{e x t}$, anisotropy factor $g$, probability of light absorption $p$ ) with other Mie calculators [28] for a variety of grain sizes [29]. Particular comparisons with experimental data have also been carried out for $\mathrm{Gd}_{2} \mathrm{O}_{2} \mathrm{~S}: \mathrm{Tb}$ phosphor material [26].

However, matter of fact, most of the times the construction of the real powder layers has remained trade secrets from the manufacturers and comparison with experimental measurements (apart from the weakness of different experimental set-ups to re-produce identical results for similar samples) is always subject of various types of uncertainties on the material synthesis, development and construction, such as: (i) the precise concentration of activator dopant, (ii) the type of the binding material and the incorporation of unknown additional substances (e.g., absorbing dyes), (iii) the exact particle size and shape, etc.

\section{Appendix A: brief physics of the simulation example}

The geometry of the simulation model considers a phosphor layer modeled as a three-dimensional powder material consisting of uniformly distributed particles (phosphor grains of equal size) of $\mathrm{Gd}_{2} \mathrm{O}_{2} \mathrm{~S}$ luminescent material (case 27 of the list of materials) embedded within a binding medium $\left(\mathrm{Na}_{2} \mathrm{SiO}_{3}\right)$. The geometry of the layer contains (a) phosphor dimensions: (i) surface dimensions: $1 \mathrm{~cm}$ (length) and $1 \mathrm{~cm}$ (width), (ii) number of pixels of surface area: 127 and (iii) layer thickness: $200 \mu \mathrm{m}$; (b) structural properties: (i) density: $7.3 \mathrm{~g} / \mathrm{cm}^{3}$, (ii) packing density: $50 \%$, and (iii) particle size: $7 \mu \mathrm{m}$, (c) optical properties: (i) light wavelength: $545 \mathrm{~nm}$, (ii) refractive index of the phosphor grains: (real part: 2.3, imaginary part: $10^{-5}$ ), (iii) refractive index of the surrounding medium: 1.35. The phosphor layer surface was assumed to be irradiated by a pencil monoenergetic X-ray beam with energy $20 \mathrm{keV}$. This energy corresponds to mass attenuation coefficient $36.88 \mathrm{~cm}^{2} / \mathrm{g}$ for $\mathrm{Gd}_{2} \mathrm{O}_{2} \mathrm{~S}$ and determines the distribution of the X-ray photon absorbed (i.e., the interaction sites) and, therefore, the distribution of light photons produced $\left(10^{6}\right.$ light photons were chosen) per depth of interaction within the layer. The Monte Carlo simulation involves the following steps:

\subsection{Step A: X-ray attenuation}

To simulate the X-ray transport within the phosphor layer, the model initially determines the free path length (fpl) of each X-ray quantum of energy $E$, according to the following expression:

$\mathrm{fpl}=\frac{1}{\mu(E) \rho \rho_{P}} \ln R$

where $\mu(E)$ is the mass attenuation coefficient of the phosphor material, $\rho$ is the bulk density, and $\rho_{P}$ is the packing density.

\subsection{Step B: X-ray to light conversion and diffusion}

For each X-ray photon interaction event leading to energy deposition, light photons are produced following an isotropic spatial distribution. After their creation, light photons are transmitted through material towards the layer surfaces according to the following stages:

\subsubsection{First stage: light travel until interaction}

Light photon travels a free path length and afterwards interacts with a phosphor particle. This distance determined according to the following equation:

$\mathrm{fpl}=\frac{1}{m_{\mathrm{ext}}} \ln R$

where $R$ is a random number uniformly distributed in the interval $(0,1]$ and $m_{\text {ext }}$ is the Mie light extinction coefficient which is computed by the following formula:

$m_{\mathrm{ext}}=V_{\mathrm{d}} A Q_{\mathrm{ext}}$

where $V_{\mathrm{d}}$ is the volume density of the phosphor material, $A$ is the geometrical cross-section of the grain and $Q_{\text {ext }}$ is the extinction efficiency factors. For the presented geometry, the $m_{\text {ext }}$ coefficient was evaluated equal to $0.216 \mu \mathrm{m}^{-1}$.

\subsubsection{Second stage: light interaction}

Light photon is either absorbed or scattered. At the site of light photon interaction, the type of interaction is expressed by the probability of light absorption $p$. This probability is calculated using the Mie light absorption and scattering coefficients $m_{\text {abs }}, m_{\text {sct }}$, as follows:

$p=\frac{m_{\mathrm{abs}}}{m_{\mathrm{abs}}+m_{\mathrm{sct}}}$

For a random number $R$, if $R \leq p$ light photon is absorbed and the photon track within the material terminates, otherwise if $R>p$ light photon is scattered and the photon track continues in a new direction. For the presented geometry, the $p$ coefficient was evaluated equal to $0.08111 \%$. 

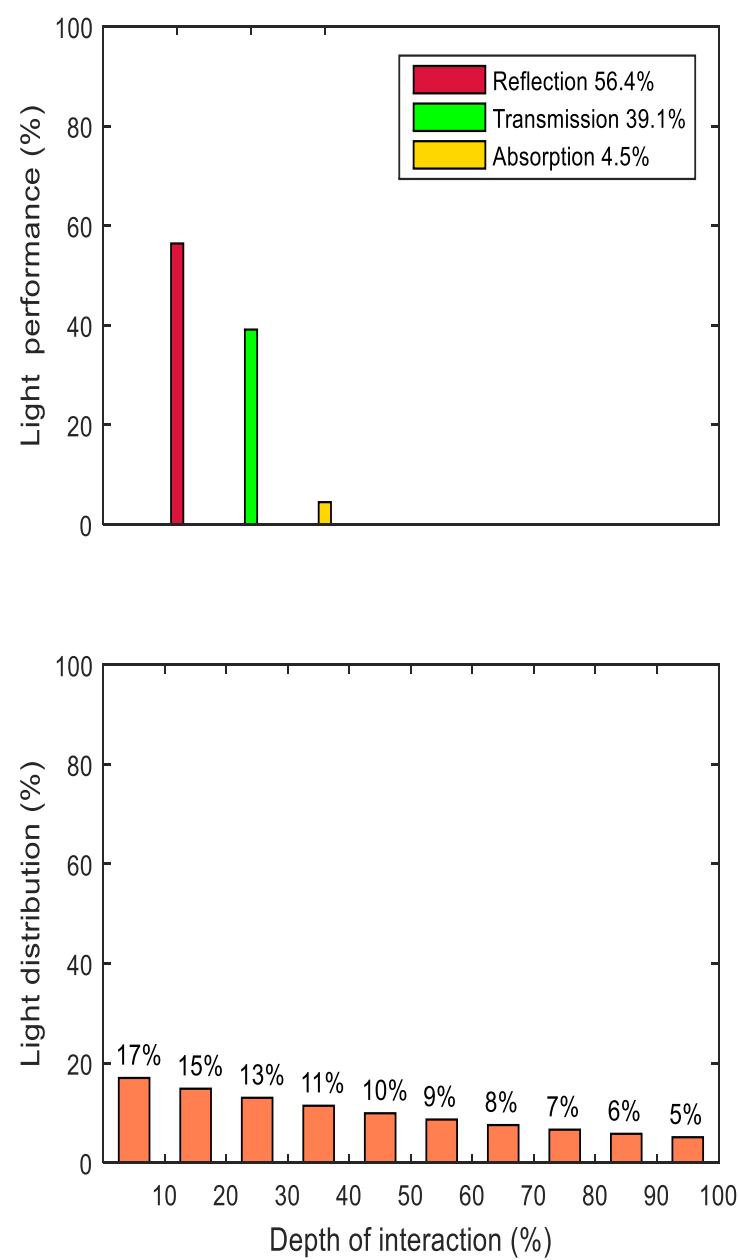

Fig. 1 Figure illustrates four separate subplots of the: (i) light performance which contains: a the percentage of light photons reflected at the upper side of the layer, $\mathbf{b}$ the percentage of light photons transmitted at the underside of the layer, and $\mathbf{c}$ the percentage of light photons absorbed within the material, (ii) distribution of light photons per

\subsubsection{Third stage: light scattering}

In case of scattering, the new direction of light photon is determined from the Henyey-Greenstein distribution, as given below:

$\cos \theta=\frac{1}{2 g}\left[1+g^{2}-\left(\frac{1-g^{2}}{1-g+2 g R}\right)^{2}\right.$ when $\left.g \neq 0\right]$

The free parameter $g$ is the anisotropy factor, which implies isotropic distribution of light for $g=0$ and sharply forward direction of light for $g=1 . R$ is a random number uniformly distributed in the interval $(0,1]$. The anisotropy factor was calculated using the following equation:
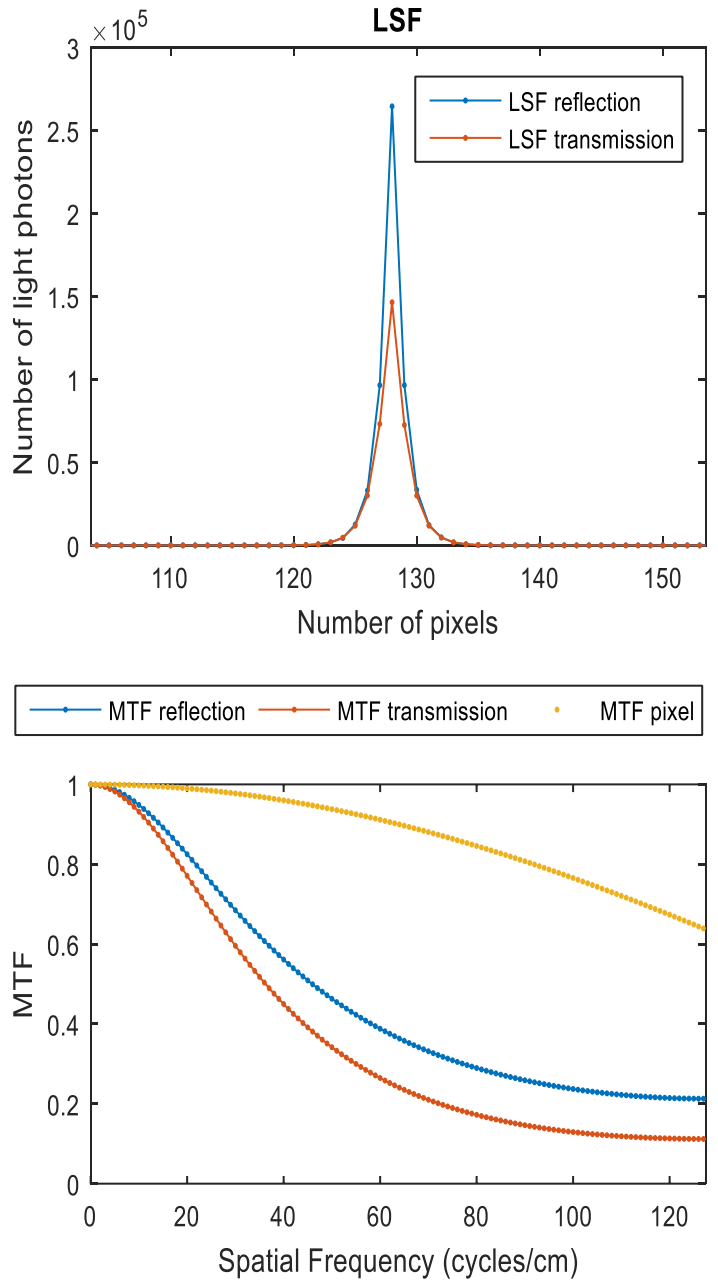

depth of interaction within the layer, (iii) LSF for reflection and transmission mode and (iv) MTF for reflection and transmission mode as well as the so-called pixel-MTF due to the pixel size effect. The setting parameters are provided in the Appendix A and Appendix B sections

$g=\frac{\int_{0}^{\pi} 2 \pi S_{11}(\theta) \cos \theta \sin \theta d \theta}{\int_{0}^{\pi} 2 \pi S_{11}(\theta) \sin \theta d \theta}$

where $S_{11}(\theta)$ is the first element of the so-called Mueller matrix, which implies that light extinction is independent of the light polarization state. For the presented geometry, the $g$ factor was evaluated equal to 0.736 . Results are illustrated in Fig. 1 which includes the following subplots: (i) the light performance, (ii) distribution of light photons per depth of interaction within the layer, (iii) LSF for reflection and transmission mode and (iv) MTF for reflection and transmission mode as well as the so-called pixel-MTF due to the pixel size effect. 
5 Appendix B: interface of the simulation example

WELCOME TO LIGHTAWE - START THE SIMULATION

\section{LAYER SETTINGS}

SET THE X-AXIS DIMENSION OF THE LAYER IN CENTIMETERS

CHOOSE BETWEEN 0.1 - 10

X-AXIS (CENTIMETERS): 1

SET THE Y-AXIS DIMENSION OF THE LAYER IN CENTIMETERS

CHOOSE BETWEEN 0.1 - 10

Y-AXIS (CENTIMETERS): 1

18 SET THE THICKNESS OF THE LAYER IN MICROMETERS

19 CHOOSE BETWEEN 1 - 1000

20 THICKNESS (MICROMETERS):100

SET THE NUMBER OF PIXELS OF THE IMAGE (LAYER OUTPUT)

CHOOSE 64 - 512

NUMBER OF PIXELS: 127

\section{STRUCTURAL PROPERTIES OF THE MATERIAL}

SET THE PARTICLE DIAMETER IN NANOMETERS

CHOOSE BETWEEN 10 - 20000

PARTICLE DIAMETER(NANOMETERS):7000

SET THE PARTICLE PACKING

CHOOSE BETWEEN 0.10 - 0.90

PACKING DENSITY: 0.50

OPTICAL PROPERTIES OF THE MATERIAL

SET THE LIGHT WAVELENGTH IN NANOMETERS

CHOOSE BETWEEN 400 - 700

LIGHT_WAVELENGTH (NANOMETERS):545

SET THE REFRACTIVE INDEX OF THE MATERIAL - REAL PART

CHOOSE BETWEEN 1.5 - 2.5 
45 REFRACTIVE INDEX OF THE MATERIAL (REAL PART):2.3

46 SET THE REFRACTIVE INDEX OF THE MATERIAL - IMAGINARY PART

47 CHOOSE BETWEEN 10^ -6 - 10^^${ }^{\wedge} 4$

48 REFRACTIVE INDEX OF THE MATERIAL (IMAGINARY PART): $10^{\wedge}-5$

SET THE REFRACTIVE INDEX OF THE MEDIUM (BINDER)

50 CHOOSE BETWEEN 1.0 - 1.5

51 REFRACTIVE INDEX OF THE MEDIUM (BINDER):1.35

\section{LIGHT BEAM PROPERTIES}

SET THE NUMBER OF LIGHT PHOTONS

CHOOSE BETWEEN $1-1^{\wedge} 7$

NUMBER OF LIGHT PHOTONS:1000000

SET THE OPTION OF SIMULATION

CASE 1: FIXED LIGHT POINT SOURCE

CASE 2: DISTRIBUTION OF LIGHT SOURCES - X-RAY PENETRATION

CHOOSE 1 OR 2

CASE:2

SET THE X-RAY ENERGY (keV)

CHOOSE BETWEEN 10 - 100

X-RAY ENERGY (keV):20

SET THE MATERIAL COMPOSITION

CHOOSE BETWEEN 1 - 62

CASE 1: Ba4Ce3F17

CASE 2: $\mathrm{BaCl}$

CASE 3: BaF2

CASE 4: BaHfO3

CASE 5: BaLu2F8

82 CASE 6: BaThF6

83 CASE 7: BaY2F8

84 CASE 8: Ba4Y3F17

85 CASE 9: Bi4Ge3O12 (BGO)

86 CASE 10: CaHfO3

87 CASE 11: CaWO4

88 CASE 12: CeCl3

89 CASE 13: CeF3

90 CASE 14: CsF 


\begin{tabular}{|c|c|}
\hline 91 & CASE 15: CsI \\
\hline 92 & CASE 16: CsGd2F7 \\
\hline 93 & CASE 17: Cs2LiYCl6 \\
\hline 94 & CASE 18: Cs3LuCl6 \\
\hline 95 & CASE 19: Cs3Lu2C19 \\
\hline 96 & CASE 20: Cs2NaCeCl6 \\
\hline 97 & CASE 21: Cs2NaLaCl6 \\
\hline 98 & CASE 22: Cs2NaLuCl6 \\
\hline 99 & CASE 23: GdAIO3 (GAP) \\
\hline 100 & CASE 24: GdBr3 \\
\hline 101 & CASE 25: GdBO3 \\
\hline 102 & CASE 26: Gd3Ga5O12 \\
\hline 103 & CASE 27: Gd2O2S (GOS) \\
\hline 104 & CASE 28: Gd2SiO5 (GSO) \\
\hline 105 & CASE 29: Gd3Sc2Al3O12 \\
\hline 106 & CASE 30: K2LaCl5 \\
\hline 107 & CASE 31: LaBO3 \\
\hline 108 & CASE 32: LaB3O6 \\
\hline 109 & CASE 33: LaBr3 \\
\hline 110 & CASE 34: LaCl3 \\
\hline 111 & CASE 35: LaF3 \\
\hline 112 & CASE 36: LaLuO3 \\
\hline 113 & CASE 37: LiBaF3 \\
\hline 114 & CASE 38: LiLuF4 \\
\hline 115 & CASE 39: LiLuSiO4 \\
\hline 116 & CASE 40: LiYF4 \\
\hline 117 & CASE 41: LuAIO3(LuAP) \\
\hline 118 & CASE 42: Lu3Al5O12 \\
\hline 119 & CASE 43: Lu2O3 \\
\hline 120 & CASE 44: LuBO3 \\
\hline 121 & CASE 45: LuBr3 \\
\hline 122 & CASE 46: LuCl3 \\
\hline 123 & CASE 47: LuF3 \\
\hline 124 & CASE 48: LuPO4 \\
\hline 125 & CASE 49: Lu2S3 \\
\hline 126 & CASE 50: Lu2SiO5 (LSO) \\
\hline 127 & CASE 51: Lu2Si2O7 \\
\hline 128 & CASE 52: NaI \\
\hline 129 & CASE 53: RbGd2Br7 \\
\hline 130 & CASE 54: RbGd2Cl7 \\
\hline 131 & CASE 55: PbWO4 \\
\hline 132 & CASE 56: YAIO3 (YAP) \\
\hline 133 & CASE 57: Y3AI5O12 (YAG) \\
\hline 134 & CASE 58: YBO3 \\
\hline 135 & CASE 59: Y2O3 \\
\hline 136 & CASE 60: Y2SiO5 (YSO) \\
\hline
\end{tabular}




\section{CASE 61: YTaO4 \\ 138 CASE 62: ZnS}

139

MATERIAL COMPOSITION - CASE:27

141

142

$$
\text { SET THE DENSITY OF THE MATERIAL (g/cm^3) }
$$

MATERIAL DENSITY (g/cm^3):7.3

144

\section{LIGHT EXTINCTION COEFFICIENT $\left(\mu \mathrm{m}^{\wedge}-1\right)$ :}

0.216

\section{OUTPUT DATA - START}

\subsection{1}

COMPUTATION TIME(MIN)

\subsection{1}

162

$$
\text { OUTPUT DATA - END }
$$

\section{References}

1. M. Hoheisel, Review of medical imaging with emphasis on X-ray detectors. Nucl. Instr. Method Phys. Res. Sect. A 563, 215-224 (2006)

2. J.C. Dainty, R. Shaw, Image science (Academic, New York, 1974)

3. J.D. Gaskill, Linear systems, fourier transforms, and optics (Wiley, New York, 1978)

4. A.P. Gibson, J.C. Hebden, S.R. Arridge, Recent advances in diffuse optical imaging. Phys. Med. Biol. 50, R1-R43 (2005)

5. S.M.W.Y. van de Ven, S.G. Elias, M.A.A.J. van den Bosch, P. Luijten, W.T.M. Mali, Optical imaging of the breast. Cancer Imaging 8, 206-215 (2008)

6. A.P. Dhawan, B. D'Alessandro, X. Fu, Optical imaging modalities for biomedical applications. IEEE Rev. Biomed. Eng. 3, 69-92 (2010)

7. T. Durduran, R. Choe, W.B. Baker, A.G. Yodh, Diffuse optics for tissue monitoring and tomography. Rep. Prog. Phys. 73, 076701 (2010)

8. M.A. Calin, S.V. Parasca, R. Savastru, M.R. Calin, S. Dontu, Optical techniques for the noninvasive diagnosis of skin cancer. Cancer Res Clin Oncol 139, 1083-1104 (2013)
9. Z. Chen, Y. Zhou, J.-T. Shen, Exact dissipation model for arbitrary photonic Fock state transport in waveguide QED systems. Opt. Lett. 42, 887-890 (2017)

10. Z. Chen, Y. Zhou, J.-T. Shen, Entanglement-preserving approach for reservoir-induced photonic dissipation in waveguide QED systems. Phys. Rev. A 98, 053830 (2018)

11. N. Metropolis, The beginning of the Monte Carlo method. Los Alamos Sci. 15, 125-130 (1987)

12. D.W.O. Rogers, 50 years of Monte Carlo simulations for medical physics. Phys. Med. Biol. 51, R287-R301 (2006)

13. M. G. Pia, T. Basaglia, Z. W. Bell, P. V. Dressendorfer, The impact of Monte Carlo simulation: a scientometric analysis of scholarly literature, Joint International Conference on Supercomputing in Nuclear Applications and Monte Carlo 2010 (SNA + MC2010), Tokyo, Japan, October 17-21, (2010)

14. G. Mie, Beitrage zur Optik tr"uber Medien, speziell kolloidaler Metallosungen. Annal. Physik 25, 377-445 (1908)

15. L.G. Henyey, J.L. Greenstein, Diffuse radiation in the galaxy. Astrophys. J. 93, 70-83 (1941)

16. H.C. van de Hulst, Light scattering by small particles (Wiley, New York, 1957) 
17. C.F. Bohren, D.R. Huffman, Absorption and scattering of light by small particles (Wiley, New York, 1983)

18. P.F. Liaparinos, Optical diffusion performance of nanophosphorbased materials for use in medical imaging. J. Biomed. Opt. 17, 126013 (2012)

19. H. Chander, Development of nanophosphors. Mater. Sci. Eng. 49, 113-155 (2005)

20. P. Moriarty, Nanostructured materials. Rep. Prog. Phys. 64, $297-$ $381(2001)$

21. L. Melcher, J.S. Schweitzer, Cerium-doped lutetium oxyorthosilicate: a fast, efficient new scintillator. IEEE Trans. Nucl. Sci. 39, 502-505 (1992)

22. A. Kienle, Anisotropic light diffusion: an oxymoron? Phys. Rev. Let. 98, 218104 (2007)

23. National Research Council, Atomic, molecular, and optical science: an investment in the future (The National Academies Press, Washington, DC, 1994)

24. W.L. Dunn, J.E. Fernández, M.I. Lopes, D.A. Bradley, J.C. Carvalho, M. Cooper, J. Pinto da Cunha, R.P. Gardner, F. Salvat, E. Sartori, A. Sood, P. Vaz, Organizing committee, workshop on use of Monte Carlo techniques for design and analysis of radiation detectors (ISRP10), 15-17 September, Coimbra, Portugal (2006)

25. I.A. Cunningham, Applied linear-systems theory in handbook of medical imaging, in Physics and psychophysics, ed. by J. Beutel, H.L. Kundel, R.L. Van Metter (SPIE, Bellingham, 2000), pp. 82-157

26. P. Liaparinos, I. Kandarakis, D. Cavouras, H. Delis, G. Panayiotakis, Modeling granular phosphor screens by Monte Carlo methods. Med. Phys. 33, 4502-4514 (2006)
27. H. Du, Mie-scattering calculation. Appl. Opt. 43, 1951-1956 (2004)

28. S. A. Prahl, Mie Scattering Calculator, Portland, OR, Oregon Medical Laser Center (2006) [Online]. Available: http://omlc. ogi.edu/calc/mie calc.html

29. P. Liaparinos, I. Kandarakis, Investigating the optical diffusion capabilities of nanophosphors for use in medical imaging (SPIE, Florida, 2013), p. 8668

30. C.W.E. van Eijk, Inorganic-scintillator development. Nucl. Instrum. Methods. Phys. Res. A. 460, 1-14 (2001)

31. C.W.E. van Eijk, Inorganic scintillators in medical imaging. Phys. Med. Biol. 47, R85-R106 (2002)

32. S. Derenzo, M. Boswell, M. Weber, K. Brennan, Data base displaying the scintillation properties of phosphor materials, (2017), created by: http://scintillator.lbl.gov/

33. J.H. Hubbell, P.N. Trehan, N. Singh, B. Chand, D. Mehta, M.L. Garg, R.R. Grag, S. Singh, S. Purl, A review, bibliography, and tabulation of KL and higher atomic shell X-ray fluorescence yields. J. Phys. Chem. Ref. Data 23, 339-364 (1994)

34. J. B. Kortright, A. C. Thompson, X-ray emission energies in X-ray data booklet, Lawrence Berkeley National Laboratory, University of California, Berkeley, United States, pp 1-8 (2009)

Publisher's Note Springer Nature remains neutral with regard tojurisdictional claims in published maps and institutional affiliations. 